\title{
Shifting Bragg peaks of cuprate stripes as possible indications for fractionally charged kinks
}

\author{
Marco Bosch, Wim van Saarloos, and Jan Zaanen \\ Instituut-Lorentz, Universiteit Leiden, P.O. Box 9506, NL-2300 RA Leiden, The Netherlands
}

(Received 12 April 2000; published 25 January 2001)

\begin{abstract}
Inspired by the recent observation that cuprate stripes tilt away from the crystal axis, we propose a new type of stripe phase in the large doping regime. This phase corresponds with a doped version of the $x<\frac{1}{8}$ stripes. The topological excitations associated with this doping are fractionally charged kinks that make the stripes fluctuate and tilt. The experimental doping dependence of the tilt angle can be used to determine the fundamental charge quantum of the stripes.
\end{abstract}

DOI: 10.1103/PhysRevB.63.092501 PACS number(s): 74.72. $-\mathrm{h}$, 74.25.Jb, 71.27.+a, 75.10. - b

In the last few years, overwhelming experimental evidence has accumulated to show that the holes in the cuprate materials, such as the high- $T_{c}$ superconductors, form various types of striped phases. ${ }^{1-3}$ The building blocks of such phases consist of domain walls, which can be viewed as stringlike configurations of holes that separate hole-free antiferromagnetic domains. Important information about the microscopic origin of stripes is contained in the doping dependence of the inverse of the stripe spacing, shown in Fig. 1(a). This spacing can be obtained directly from the distance $\delta$ between the measured Bragg peaks [Fig. 1(b)]. It was experimentally found that $\delta$ is, to a good approximation, directly proportional to doping $x$ for a variety of stripe systems like the nickelates ${ }^{4}$ and manganites. ${ }^{5}$ It is also found in cuprate stripe phases, but only in the doping regime where $x$ $\lesssim \frac{1}{8}$, while for $x \gtrsim \frac{1}{8}, \delta$ becomes roughly $x$ independent; see Fig. 1(a). ${ }^{6,7}$ Quite recently it was found ${ }^{8-10}$ that in a number of cuprates the spin peaks tilt away from the $(1,0)$ or $(0,1)$ axis in reciprocal space. This puzzling behavior corresponds with a tilt of the stripe phase away from the main crystal axis of the perovskite planes in real space and seems characteristic for the $x \gtrsim \frac{1}{8}$ regime. We want to suggest here that this tilt might reflect the presence of fractionally charged solitons living on the stripes. This notion leads to an explicit relation between the $\delta$ versus $x$ dependence and the $Y$ shift, which, if confirmed experimentally, allows one to determine the topological charge of stripes.

The $x \lesssim \frac{1}{8}$ stripes might be viewed as the condensation of the topological defects associated with doping the half-filled Mott insulator $^{1-14}$ [see Fig. 2(a)]. We suggest here that the $x \gtrsim \frac{1}{8}$ state should be viewed in turn as the condensation of the topological defects associated with doping the stripe state itself. This state is as indicated in Fig. 2(b). The stripes for $x \gtrsim \frac{1}{8}$ are locally not different from the $x \lesssim \frac{1}{8}$ stripes sketched in Fig. 2(a), except that once in a while the stripe "steps sideways," it forms kinks. These kinks carry half the fundamental charge quantum associated with the stripe insulator of the $x \lesssim \frac{1}{8}$ regime and as they move a stripe always in the same direction they cause the orientation of the stripe phase to deviate from the lattice axis, explaining the $Y$ shift.

Our central assumption is that the cuprate stripe phase in the $x \lesssim \frac{1}{8}$ regime is insulating on the microscopic scale. Experimentally, the cuprate stripe phases are metalliclike and a popular viewpoint is that the electronic state on the stripes is metallic. However, the $\delta=S x$ relation, where $S$ is the linear slope in the small $x$ limit, argues strongly against this internal metallicity. Its meaning, already suggested by earlier meanfield calculations, is simply that every hole stabilizes a piece of charged stripe with a length that is an integer multiple of the lattice constant. This special stability, when the electron charge and the lattice are commensurate, shows that the internal electronic state of a stripe is insulating on short length scales. At the same time, stripes that are internally insulating on microscopic scales are not necessarily inconsistent with metallic behavior on macroscopic scales. The stripe metal

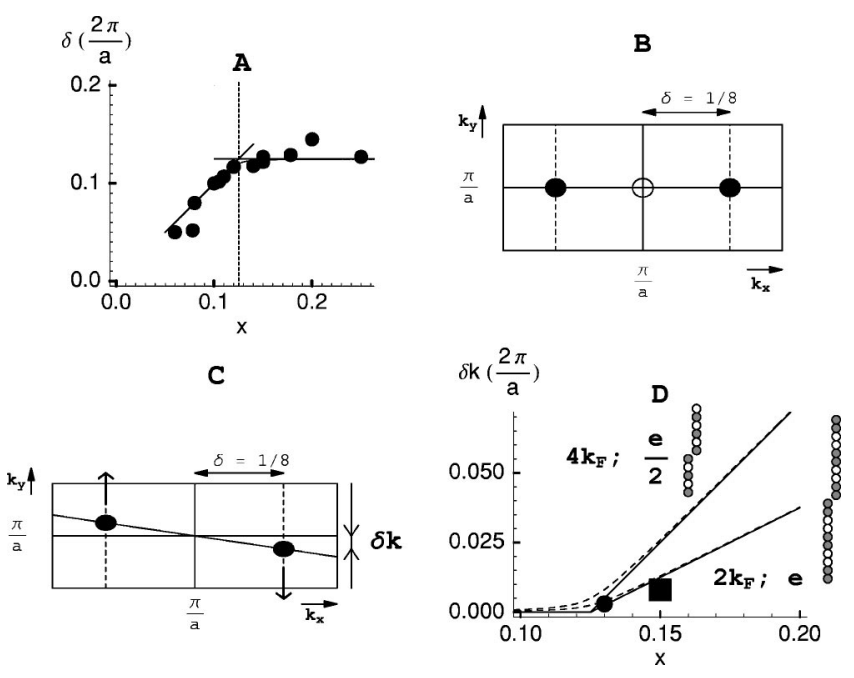

FIG. 1. (a) The inverse distance $\delta$ between the stripes as a function of doping $x$ as measured by Yamada et al. (Ref. 6). For $x$ $<0.05$, stripes are diagonal. The straight lines are for a sharp transition at $x=1 / 8$, the curve for a smooth crossover. (b) Sketch of the position of the incommensurate peaks in Fourier space as measured by neutron scattering. The open circle shows where the peaks would have been if the system would have been a commensurate antiferromagnet. We only show the peaks resulting from one layer. (c) We predict a shift $\delta k$ of the Fourier peaks caused by the existence of kinks in the stripes. Because of the resulting tilting of the unit cell, the peaks would no longer be on the axis, but move along the dashed lines. The horizontal distance to the $(\pi / a, \pi / a)$ position stays at $\delta=\frac{1}{8}$. (d) Prediction of the magnitude of the shift $\delta k$ as a function of $x$ for two possible kinds of stripes: $4 k_{F}$ stripes with kinks with fractional charge $e / 2$ and $2 k_{F}$ stripes with kinks with charge $e$. The curves belong to the same smooth crossover as the one shown in Fig. 1(a). Experimental points: Matsushita et al. (Ref. 8); $\square$ Lee et al. (Ref. 9). 
A

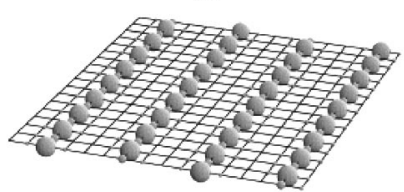

C

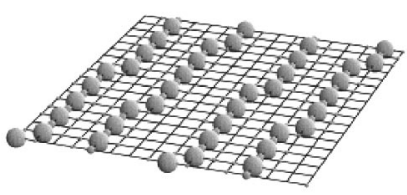

B

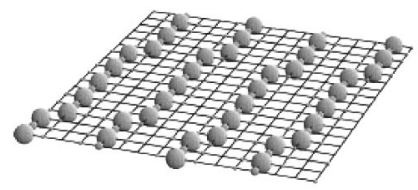

D

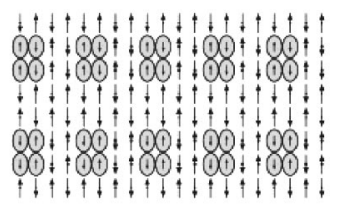

FIG. 2. (a) A system of half-filled, site-centered $4 k_{F}$ stripes with doping $x=\frac{1}{8}$ as calculated in a mean-field Hubbard model. The radius of the spheres denotes the hole density at a site. The spin density is not shown. (b) The same kind of stripe, but now at a doping of $x=0.139$, so $x$ is bigger than $\frac{1}{8}$. This system has four more holes than the system in (a). The four extra holes are localized in eight, kinks, so every kink has a fractional charge of $e / 2$. (c) The same system as (b), only now the Coulomb repulsion has been reduced by making a Wigner crystal. (d) A system of two halffilled, bond-centered $2 k_{F}$ stripes. The radius of the circles gives the hole density; the length of the arrows gives the local magnetization. This figure is very similar to a recent DMRG calculation by White and Scalapino ${ }^{14}$ on a $t-J$ model. Notice that every cluster of four big circles contains in total approximately two holes, with a singlet orientation for the spins: real-space Cooper pairs.

might correspond with a quantum-disordered stripe insulator, characterized by a growth of the quantum fluctuations when length and time scales are increasing. Although we will not address this issue in any further detail, the remainder should be read as a suggestion for a critical experimental test of this hypothesis.

We follow the literature with regard to the nature of the reference insulator in the $x \lesssim \frac{1}{8}$ regime. ${ }^{15,16}$ The slope $S$ of the $\delta$ versus $x$ relationship for small $x$ implies that one hole stabilizes two charge stripe unit cells. Accordingly, the dynamics associated with the electrons along the stripe can be associated with that of a quarter filled one-dimensional (1D) fermion system. ${ }^{17}$ It is well known that such a system can become Mott insulating by breaking translational symmetry with real-space charge periods $2 a$ and $4 a$, or wave vectors $4 k_{F}$ and $2 k_{F}$, respectively, where $k_{F}=\pi /(4 a)$ ( $a$ is the lattice constant). Schematically, the ordering pattern on the

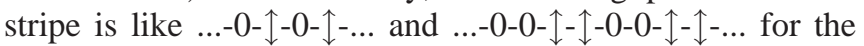
$4 k_{F}$ and $2 k_{F}$ stripes, respectively. Here 0 denotes the hole and $\uparrow$ the presence of a spin, which we expect to be disordered due to quantum fluctuations. At present it is not known which type of density wave order is realized on the stripe. All that really matters for the remainder of our discussion is that the $4 k_{F}$ stripe should be considered as the condensation of the charge $e$ of one hole, ${ }^{15}$ while the $2 k_{F}$ stripe is associated with the charge quantum of a pair of holes $2 e .^{16}$

Let us now turn to the $x \gtrsim \frac{1}{8}$ regime. The (near) independence of $\delta(x)$ as a function of doping $x$ implies that a fraction of the holes $x^{\prime}=x-\delta(x) / S \approx x-\frac{1}{8}$ cannot be "absorbed" by the $x \lesssim \frac{1}{8}$ insulator. These excess holes should dope the $x \lesssim \frac{1}{8}$ state and it is expected that these holes dope

A

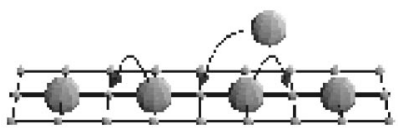

C
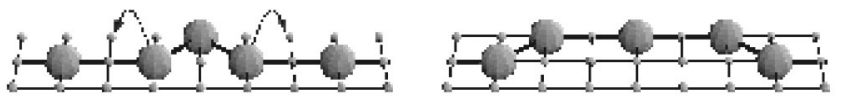

FIG. 3. Soliton dynamics in a strongly coupled doped $4 k_{F}$ stripe. The small spheres denote the lattice points with one electron, the large spheres are holes. The spins are not shown. (a) The reference state: localized stripe with $4 k_{F}$ charge-density wave, onto which we add an extra hole. (b) If the stripe is rigid, the doped hole separates in a left- and right-moving soliton, both carrying half the electron charge. (c) When the curvature energy becomes less than the charge compressibility energy, the hole can escape "sideways." (d) As a result, the stripe now has two kinks that can move independly of each other.

the "soft" insulator associated with the density wave on the charge stripes instead of the magnetic domains, the remnants of the "hard" insulator of half filling. Doped charge-density wave states are well understood in the context of conventional 1D systems. ${ }^{18}$ A key concept was introduced by Schrieffer ${ }^{19}$ in the study of polyacetyleen: the elementary excitations in such a system are not electrons but in fact parts of an electron. The reason is that these excitations can be viewed as electrons bound to topological defects in the density wave order parameter. That the charge of the electron fractionalizes in doped $4 k_{F}$ density wave systems is easily seen by considering the strong coupling limit. ${ }^{20}$ The undoped state with one (static) hole added at the central site is indicated in Fig. 3(a). After a couple of hops a configuration is reached where the bare hole has decayed into two propagating excitations carrying half the hole charge $(e / 2)$, which are at the same time domain walls (kinks) in the density wave [Fig. 3(b)]. Such an e/2 domain wall corresponds with a region of enhanced charge density, and hence an increased Coulomb energy. In contrast to a one-dimensional crystal, a stripe has the additional freedom of moving sidewards, thereby increasing the distance between the two holes associated with the domain wall, ${ }^{20}$ as sketched in Fig. 3(c). Thus, the fractionally charged domain walls also cause the stripe to step sidewards, modulating the position of the stripes in space, see Fig. 3(d). If all the kinks are in the same direction, the net result is that the stripe takes on average an orientation in space that is tilted away from the lattice axis, very much like slanted phases that are found in lattice string models. ${ }^{21}$

Let us now consider a dense system of such "slanted" stripes. In the presence of any stripe-stripe interaction this will condense at zero temperature in a stripe phase where all the stripes are tilted in the same direction, our explanation for the $Y$ shift. It is also expected that the kinks themselves order in a regular pattern at zero temperature. The argument is the usual one: the kink gas on a single stripe becomes at long wavelength a Luttinger liquid showing algebraic longrange order. In the presence of any interaction between the Luttinger liquids on different stripes this will change into 
true long-range order at zero temperature. ${ }^{22}$

The above considerations do not depend on the type of stripe density wave order, except that the charge $q$ of the kinks is different. How should the charge of the kinks be counted for $2 k_{F}$ parent stripes? The rule is that the charge of the soliton is half the charge quantum associated with the parent. Since the $2 k_{F}$ stripes can be considered as condensations of pairs of electrons, the charge associated with a charged kink in the $2 k_{F}$ stripe becomes one electron charge. In terms of the strong-coupling cartoon of Fig. 3, one should now associate two sites of the real lattice with one site in the lattice of Fig. 3(c) so that Fig. 3(d) can be mapped back to the $2 k_{F}$ case by inserting the doubled lattice and the doubled charges.

These general ideas can be illustrated with explicit calculations. Since we are considering fully ordered insulating states, mean-field theory is able to provide meaningful qualitative outcomes. The reference $2 k_{F}$ and $4 k_{F}$ stripe insulators actually both exist in the Hartree-Fock solutions of the Hubbard model as low-lying, but weakly metastable states: the true mean-field ground state corresponds with filled stripes (one hole per stripe unit cell). ${ }^{16,23}$ In Fig. 2(d) we show a typical example of a straight bond-centered $2 k_{F}$ stripe, calculated with the mean-field approximation, which strongly resembles the stripe patterns found in the density-matrix renormalization-group calculations by White and Scalapino for the $t$ - $J$ model. ${ }^{24}$

By constraining the distance between the stripes to be fixed, increasing the hole density, and using appropriate boundary conditions, doped stripes can also be investigated in mean-field theory. We find that such doped stripes, if they exist as locally stable mean-field solutions, prefer localized fractionally charged kinks. A typical example is shown in Fig. 2(b), where the density of excess holes is indicated for a $4 k_{F}$ stripe state at a hole density of $x=0.139$. In line with the qualitative arguments given above, every hole doped into the $4 k_{F} x=\frac{1}{8}$ reference insulator gives rise to two kinks, each carrying a charge of $e / 2$ and moving the stripe sideways by one lattice constant. Although these "slanted" stripes are quite stable solutions if the reference insulator is of the $4 k_{F}$ variety, we did not manage to find stable solutions for the $2 k_{F}$ stripes doped with additional holes-within mean-field theory, such kinks tend to disintegrate.

In order to get some feeling of the relative stability of the $4 k_{F}$ slanted phase we compared it with a straight stripe where an additional hole is simply inserted in the straight density wave (...- $-0-0-0-\uparrow \ldots)$. On the Hartree-Fock level, a stripe with two kinks of charge $e / 2$ always has significantly lower energy (of the order $0.01 t$ ). We also checked that the sidesteps can be further stabilized by including a nearestneighbor Coulomb interaction. The energetics associated with the relative transversal orientation of the kinks is a more delicate matter that will strongly depend on the distance between kinks. We believe that collective fluctuation effects will promote long-range slanted order over zigzag-type stripe patterns, in much the same way as quantum fluctuations make lattice strings directed. ${ }^{21}$

Of course, our calculations merely serve to illustrate the principle - neither the question of which possibility is real- ized in nature nor the question of whether the "topological", insulator is the proper reference state can be settled by these calculations. However, simple predictions follow, which at least in principle can be checked by experiment. Most importantly, the charged kinks offer an explanation for the $Y$ shift that has already been observed in experiments. However, the kink notion implies that the superlattice peaks of the slanted stripe phase are located along the straight lines crossing the superlattice peaks associated with the $1 / 8$ phase as indicated in Fig. 1(c). The reason is that the average distance between the stripes does not change when the stripes are making sidesteps, thereby leaving the distance between the peaks in reciprocal space along the horizontal axis in Fig. 1(c) unchanged.

Lattice commensuration effects make a transition to kinked stripes around $x=\frac{1}{8}$ most likely. However, due to complications associated with quenched disorder we have no prediction for whether there should be a sharp transition or a rapid but smooth crossover. However, the scenario that there are no free holes, but that instead all holes go onto stripes, leads to an immediate relation between the $\delta(x)$ dependence and the $Y$ shift. Let us define the magnitude of the $Y$ shift as $\delta k$ and the fractional charge (in units of $e$ ) carried by the kink as $q$. Then $x^{\prime}=x-\delta(x) / S \approx x-\frac{1}{8}$ is the hole density associated with doping the stripes in the $x \gtrsim \frac{1}{8}$ regime. It can be shown that the $Y$ shift obeys $\delta k=x^{\prime} / 2 q 2 \pi / a$ and that $\delta$ $=\left(x-x^{\prime}\right) 2 \pi / a$, where $a$ is the lattice constant. So we have $\delta+2 q \delta k=2 \pi / a x$, independent of whether there is a crossover or sharp transition, and where it takes place. In Fig. 1(d) we show our prediction for the $Y$ shift as a function of $x$ for the $2 k_{F}$ and $4 k_{F}$ cases, both for the case of a sharp transition at $1 / 8$ and for the same smooth crossover as in Fig. 1(a). We have also indicated in this figure two experimental results for the $Y$ shift, where the $x=0.15$ point has been reported by Lee et $a{ }^{9}{ }^{9}$ for an oxygen doped sample of $\mathrm{La}_{2} \mathrm{CuO}_{4}$ and the $x$ $=0.13$ point is measured in $\mathrm{La}_{2-x} \mathrm{Sr}_{x} \mathrm{CuO}_{4}$ sample by Matsushita et al. ${ }^{8}$ There is also one experimental indication of a $Y$ shift at dopings slightly less than $\frac{1}{8}$ by Kimura et al., ${ }^{10}$ which suggests that there is a smooth crossover. Clearly an extensive experimental study is called for to establish whether the $Y$ shift is due to kinks and whether our relationship between $\delta k$ and $\delta(x)$ holds. The existing data seem to favor a kink charge of $e$ over charge $e / 2$, suggesting that the elementary charge quantum associated with the stripe phase is $2 e$. Given the proximity of a superconductor characterized by charge quantum $2 e$, such a stripe charge quantization would obviously be quite interesting.

We argued that the charged kinks themselves should order. It should therefore in principle be possible to observe the superlattice reflections of this kink lattice in diffraction experiments. As we argued, the kinks on single stripes will repel but the kink order from stripe to stripe is less easy to establish. On the one hand, the kinks carry charge and under the assumption that the screening length is of order or larger than the average kink separation, the kinks would tend to maintain a maximum separation, thereby forming a Wigner crystal as indicated in Fig. 2(c). However, one could imagine 
that elastic deformation energies are minimized when kinks line up as indicated in Fig. 2(b). The precise realization of the kink superlattice is therefore a subtle, quantitative matter.

In conclusion, inspired by the tilting of the stripes seen at higher dopings we have proposed a scenario of stripe phases characterized by a proliferation of fractionally charged soli- tons. Remarkably, by studying merely the structural characteristics of this phase that is realized at higher dopings, the elementary charge quantum numbers of the stripes can be established. This is undoubtedly a crucial piece of information in the quest for the understanding of high- $T_{c}$ superconductivity. ${ }^{15}$
${ }^{1}$ V. J. Emery, S. A. Kivelson, and J. M. Tranquada, Proc. Natl. Acad. Sci. U.S.A. 96, 8814 (1999).

${ }^{2}$ R. F. Service, Science 283, 1106 (1999).

${ }^{3}$ J. Zaanen, Science 286, 251 (1999).

${ }^{4}$ J. M. Tranquada, D. J. Buttrey, and V. Sachan, Phys. Rev. B 54, 12318 (1996).

${ }^{5}$ M. Hervieu et al., Eur. Phys. J. B 8, 31 (1999); S. Mori, C. H. Chen, and S.-W. Cheong, Phys. Rev. Lett. 81, 3972 (1998).

${ }^{6}$ K. Yamada et al., Phys. Rev. B 57, 6165 (1998).

${ }^{7}$ The special status of $x=1 / 8$ was recently confirmed by magnetotransport measurements: see T. Noda, H. Eisaki, and S. Uchida, Science 286, 265 (1999).

${ }^{8}$ H. Matsushita et al., J. Phys. Chem. Solids 60, 1071 (1999).

${ }^{9}$ Y. Lee et al., Phys. Rev. B 60, 3643 (1999).

${ }^{10}$ H. Kimura et al., Phys. Rev. B 59, 6517 (1999).

${ }^{11}$ J. Zaanen and O. Gunnarsson, Phys. Rev. B 40, 7391 (1989); J. Zaanen, J. Phys. Chem. 59, 1769 (1998).

${ }^{12}$ S. A. Kivelson and V. J. Emery, Synth. Met. 80, 151 (1996).

${ }^{13}$ L. P. Pryadko, S. A. Kivelson, V. J. Emery, Y. B. Bazaliy, and E. A. Demler, Phys. Rev. B 60, 7541 (1999).

${ }^{14}$ S. R. White and D. J. Scalapino, Phys. Rev. Lett. 81, 3227 (1998).

${ }^{15}$ C. Nayak and F. Wilczek, Phys. Rev. Lett. 78, 2465 (1997); Int. J. Mod. Phys. B 10, 2125 (1996).

${ }^{16}$ J. Zaanen and A. M. Oles, Ann. Phys. (Leipzig) 5, 224 (1996).

${ }^{17} \mathrm{In}$ recent photoemission experiments evidence is found for an underlying one-dimensional band structure characterized by a Fermi momentum of a quarter-filled system: X. J. Zhou et al., Science 286, 268 (1999).

${ }^{18}$ F. D. M. Haldane, Phys. Rev. Lett. 45, 1358 (1980).

${ }^{19}$ J. R. Schrieffer, in Proceedings of the International School of Physics "Enrico Fermi," course LXXXIX, edited by F. Bassani (Elsevier, New York, 1985); S. Kivelson, J. R. Schrieffer, Phys. Rev. B 25, 6447 (1982).

${ }^{20}$ J. Zaanen, O. Y. Osman, and W. van Saarloos, Phys. Rev. B 58, R11 868 (1998).

${ }^{21}$ H. Eskes, R. Grimberg, W. van Saarloos, and J. Zaanen, Phys. Rev. B 54, R724 (1996).

${ }^{22}$ We notice that the argument of S. A. Kivelson, E. Fradkin, and V. J. Emery, Nature (London) 393, 550 (1998) for the dephasing of density correlations on different stripes by transversal stripe fluctuations does not apply here. Although valid in the continuum, the kinks we consider are tied to the presence of an underlying crystal lattice and it is easily seen that the dephasing does not occur.

${ }^{23}$ In Gutzwiller mean field (qualitatively similar but more accurate than Hartree-Fock) half-filled stripes become the ground state of the Hubbard model with added long-range Coulomb interaction: see G. Seibold, C. Castellani, C. D. Castro, and M. Grilli, Phys. Rev. B 58, 13506 (1998).

${ }^{24}$ S. R. White and D. J. Scalapino, Phys. Rev. B 61, 6320 (2000). 